REVUE DE L'INSTITUT

FRANÇAIS D'HISTOIRE

EN ALLEMAGNE

\section{Revue de l'IFHA}

Revue de l'Institut français d'histoire en Allemagne

$1 \mid 2009$

IFHA 1

\title{
Old and New Borders in Europe
}

École d'été des Instituts Français de Recherche à l'Étranger (IFRE)

européens / International and Interdisciplinary Summer School, Berlin / Francfort-sur-l'Oder, 6-10 juillet 2009

Axelle Chassagnette, Guillaume Garner, Thomas Lienhard et Mathieu Olivier

\section{OpenEdition}

\section{Journals}

Édition électronique

URL : http://journals.openedition.org/ifha/335

DOI : 10.4000/ifha.335

ISSN : 2198-8943

Éditeur

IFRA - Institut franco-allemand (sciences historiques et sociales)

Édition imprimée

Date de publication : 30 septembre 2009

Pagination : 108-112

ISSN : 2190-0078

Référence électronique

Axelle Chassagnette, Guillaume Garner, Thomas Lienhard et Mathieu Olivier, «Old and New Borders in Europe », Revue de l'IFHA [En ligne], 1 | 2009, mis en ligne le 07 février 2013, consulté le 15 septembre 2020. URL : http://journals.openedition.org/ifha/335

Ce document a été généré automatiquement le 15 septembre 2020

(C)IFHA 


\title{
Old and New Borders in Europe
}

École d'été des Instituts Français de Recherche à l'Étranger (IFRE)

européens / International and Interdisciplinary Summer School, Berlin /

Francfort-sur-l'Oder, 6-10 juillet 2009

\author{
Axelle Chassagnette, Guillaume Garner, Thomas Lienhard et Mathieu \\ Olivier
}

\section{NOTE DE L'ÉDITEUR}

Rapport établi par Axelle Chassagnette, Guillaume Garner, Thomas Lienhard et Mathieu olivier

1 Avec le soutien de la MHFA, le Centre Marc Bloch (CMB) et l'Université Viadrina de Francfort-sur-l'Oder ont organisé du 6 au 10 juillet 2009 une école d'été internationale sur le thème des frontières européennes, anciennes et actuelles. Par sa durée et l'internationalité des intervenants, cette manifestation donna une dimension supplémentaire à la quatrième École d'été des IFRE, que le Centre Marc Bloch accueillait à cette occasion pour la deuxième fois, après la très belle édition 2007.

2 Le lundi après-midi vit la tenue d'une première session, sous la forme d'un séminaire de méthode à l'adresse des doctorants des IFRE dont les recherches n'entraient pas dans le cadre thématique retenu pour l'École d'été 2009. Il fut l'occasion de passer en revue les travaux en cours dans plusieurs des centres de recherche du réseau européen. Marine Roussillon (Maison Française d'oxford) a présenté les grandes lignes de son étude en cours sur la réception du Moyen Âge à l'âge classique en France; ce chapitre encore mal connu du médiévalisme européen donna matière à s'interroger plus largement sur l'analyse des phénomènes de réception. Entre République des Lettres et correspondance diplomatique, Alexandre Tessier (Maison Française d'Oxford) a étudié le fonctionnement d'un réseau d'informateurs au XVIIe siècle, à partir de la figure de Sir Joseph Williamson (1660-1680). Les deux dernières contributions ont propulsé l'auditoire depuis l'Ancien Régime à l'actualité brûlante. Gwénaëlle Mainsant (CMB) s'est interrogée sur la question du genre dans le contexte des enquêtes policières sur 
les réseaux de prostitution ; elle a montré comment les conceptions genrées présidaient au traitement étatique du phénomène au prisme de l'intervention policière. Anne Joly (CMB) s'est penchée sur le positionnement de la nébuleuse idéologique de la "gauche radicale " outre-Rhin à propos de la question nationale allemande à la suite de la réunification.

Le mardi 7 juillet a vu s'amorcer la réflexion commune sur la problématique frontalière en Europe. Après les très utiles remarques introductives de Béatrice von Hirschhausen et Daniel Schönpflug ( $\mathrm{CMB}$ ), plusieurs contributions, à partir d'études de cas diverses, se sont efforcées d'inscrire la problématique de la constitution des frontières dans une profondeur historique. Mathieu Olivier (MHFA) est venu donner quelques éléments sur la construction discursive des frontières prussiennes dans les chroniques médiévales de l'ordre Teutonique (XIVe-XVe siècles). Axelle Chassagnette (MFHA), riche dossier cartographique à l'appui, s'est interrogée sur les modalités et de la démarcation dans la représentation des territoires du Saint-Empire au XVIe siècle, et surtout sur les enjeux de ces limitations, pas toujours aussi évidentes que n'invite à le penser notre culture contemporaine des frontières. Ivan Sokolovski (Novosibirsk) a proposé enfin quelques pistes de réflexion sur la construction de la frontière sibérienne de la Russie au XVIIe siècle, inspirées par ses recherches sur le réseau de fortifications édifiées à mesure de la progression de la pénétration moscovite à l'est de l'Oural.

4 La réflexion s'est prolongée l'après-midi avec trois interventions portant sur les XVIIIe et XIXe siècles, et placées par conséquence sous le signe de la montée en puissance des États-nations. Benjamin Landais (Paris I) a abordé le cas d'une zone frontalière à réorganiser de fond en comble à la suite d'un processus d'extension territoriale, en l'occurrence celle des Habsbourg dans l'Europe balkanique au XVIIIe siècle. Ilsen About (Institut Européen de Florence) a posé la question de la place revenant au traitement policier de la mobilité tzigane dans l'émergence d'une culture administrative du contrôle des déplacements transfrontaliers, à l'échelle nationale et internationale. La gestion internationale des frontières fut également au cœur de l'intervention de Volker Prott (Hambourg), qui a présenté son projet de recherche sur le rôle des grandes conférences dans l'évolution des tracés frontaliers dans l'Europe au tournant des XIXe et XXe siècles.

5 Si la mise en place des États-nations est inséparable de la problématique frontalière dans l'Europe du XVIIIe au premier XIXe siècle, la bipartition du continent et la construction européenne en ont déplacé les enjeux dans le dernier demi-siècle. Villehôte des journées d'études, mais aussi ville-symbole de l'érection des nouvelles frontières de Guerre Froide, Berlin fut évoquée dans la contribution de Michelle Standley (New York), consacrée au tourisme occidental dans le Berlin-Est des années 1960-1970. Centrées sur l'Europe de l'Ouest, et éminemment complémentaires, les deux interventions d'Emmanuel Comte (Paris IV) et Marcel Berlingoff (Heidelberg) ont respectivement porté sur le processus d'ouverture des frontières à la circulation des personnes dans l'Europe communautaire des années 1950-1980, et sur le durcissement des politiques migratoires dans quelques pays choisis (France, Allemagne, Suisse) à la même époque.

6 Un dernier temps de la journée a consacré le déplacement de l'attention de la frontière aux implications de son franchissement (illégal) dans le contexte européen de ces dernières décennies. Oumar Silué (Bouaké, Côte d'Ivoire) a proposé une remise en perspective de la «forteresse Europe» du point de vue de ceux qui sont en les 
principales victimes, les candidats à l'immigration issus de l'Afrique subsaharienne. Ariadna Ripoll Servent (Brighton) a exploré les mécanismes institutionnels européens de prise de décision dans le domaine du traitement des migrants. Insa Breyer (CMB) et Stefan Le Courant (Paris) ont donné chair au vécu des populations concernées au premier chef par ces politiques, selon deux approches différentes. La première compara le ressenti des immigrés clandestins dans le contexte allemand et français; le second s'est plus particulièrement intéressé aux centres de rétention administrative en France, antichambre de l'expulsion mais aussi lieu créateur en lui-même de nouvelles frontières.

7 La troisième journée fut l'occasion d'étudier la problématique frontalière sous un angle particulier, celui des politiques environnementales actuelles. Julien Gardaix (Bordeaux) a étudié le cas des frontières maritimes à l'heure de nouveaux usages, tandis que Magdolna Prantner (Wuppertal) s'est efforcée d'évaluer l'impact des directives européennes dans l'émergence du secteur des énergies renouvelables en Europe centrale et orientale. La dernière intervention d'Inès Dombrowsky (Leipzig) comportait déjà un caractère de retour sur expérience, celle en l'occurrence de la gestion écologique transfrontalière de deux axes fluviaux, le Rhin et l'Elbe. La manifestation se transporta ensuite à Francfort-sur-l'Oder pour une dernière journée de débats. La cité et sa jumelle polonaise Słubice constituèrent un décor idéal au moment de s'interroger sur le présent et le devenir des régions frontalières européennes. C'est donc tout naturellement que cet exemple de gouvernance transfrontalière fut examiné en détail, à travers la communication de Quentin Mackre (Montpellier). Étudié par Cristina Stanculescu (Bruxelles), le cas de Timisoara, cité aux identités multiples, vint également nourrir la réflexion sur les modalités et les enjeux de collaboration bi- ou trilatérale à la mesure d'une ville. L'échelle des coopérations transfrontalières régionales ne fut pas oubliée. Monika Sonntag (Berlin) aborda les politiques culturelles impliquant acteurs luxembourgeois et autres partenaires régionaux. Mihaela Arambasa (Potsdam) brossa un tableau très évocateur de l'impact au quotidien de la frontière extérieure de l'Union européenne pour ses riverains roumains et moldaves. Sa contribution fut avantageusement complétée par celle de Mihai Bulai (Iąi) sur les réorganisations spatiales induites par la frontière nord-est de la Roumanie. La frontière extérieure de l'Union européenne et les problèmes qu'elle génère furent en outre au centre de la communication de Pascal Orcier (Lyon) sur les enjeux de voisinage dans le contexte régional des Républiques baltes. Enfin, Siarhei Liubimau (Varsovie) offrit en complément de cette riche palette d'études de cas locaux et régionaux quelques éléments de recadrage général sur les environnements frontaliers au sein de l'Union Européenne.

8 Le programme ménagea une place importante à différentes visites, ainsi qu'à la présentation de réalisations artistiques sur le thème des frontières européennes, moments de répit mais aussi prolongements captivants de la réflexion. Les participants purent ainsi assister en avant-première à la projection du film documentaire Border Speaking réalisé par Simon Brunel et Nicolas Pannetier sur la base d'entretiens conduits avec des frontaliers vivant aux confins des deux Europe séparées près de 40 ans durant par le Rideau de fer. Autre moyen de ressusciter dans les consciences des divisions qui ne sont plus, la réalité virtuelle peut également être mise à contribution pour approcher au plus près du vécu de frontière: c'est l'ambition qui anime le projet Virtuelle Berliner Mauer, présenté par Teresa Reuter. 
9 Échanges riches, marqués par un vrai dialogue interdisciplinaire, auditoire très international, atmosphère cordiale : cette "semaine d'études ", à laquelle la MHFA fut heureuse de contribuer, fut à tout point de vue une belle réussite, à mettre au crédit des organisateurs qu'il convient de remercier ici très chaleureusement. 